\title{
Efficiency of Nano-Electrospray Ionization
}

\author{
Ayman El-Faramawy, ${ }^{*}$ K. W. Michael Siu, and Bruce A. Thomson* \\ Department of Chemistry and Center for Research in Mass Spectrometry, York University, Toronto, \\ Ontario, Canada
}

The efficiency of nano-electrospray ionization, defined as the flux of ions reaching the detector of a triple-quadrupole mass spectrometer divided by the flux of analyte ions leaving the needle, has been measured in a series of controlled experiments with dodecyltrimethyl ammonium (DDTMA) bromide, myoglobin, Glu- [1]-fibrinopeptide, and gramicidin S. By varying the flow rate from each needle, the optimum efficiency was determined. In general, the efficiency increased as the flow rate decreased. For DDTMA, efficiencies of up to $12 \%$ were measured, although efficiencies of $\sim 1 \%$ were more common. Ion current measurements indicated efficient transfer of ions from the needle through to the detector. Significant needle-to-needle variations in efficiency were encountered and attributed to variations in ion-generation efficiency. (J Am Soc Mass Spectrom 2005, 16, 1702-1707) (c) 2005 American Society for Mass Spectrometry

$\mathrm{N}$ ano-electrospray ionization (nano-ESI) is generally recognized as the most efficient method of introducing a liquid sample for direct analysis by mass spectrometry. Broadly speaking, nano-ESI is a form of electrospray, with the same fundamental ionization process of droplet formation followed by multiple uneven Rayleigh divisions, and finally desorption of pre-formed ions from the droplet $[1,2]$. The technique is distinguished from more conventional forms of electrospray by the fashion in which it is carried out. One to two microliters of sample is deposited into a glass or quartz tube that has a tip diameter in the order of $1 \mu \mathrm{m}$, and is sprayed from the tip by applying a voltage to the solution. The actual flow rate is usually a few $\mathrm{nL} / \mathrm{min}$ to a few tens of $\mathrm{nL} / \mathrm{min}$, controlled by the diameter of the tip, the voltage applied, and the backpressure which is sometimes applied to the tube content. Karas et al. [3-5] have nicely shown that, in comparison with electrospray, nano-ESI reduces interference effects from salts and other species and provides better sensitivity toward a variety of analytes, including peptides and oligosaccharides, in samples contaminated by high levels of salts. They attributed this to the reduced droplet size compared with electrospray at higher flow rates.

Nano-ESI is typically used for peptide and protein analysis because of the ability to analyze a small volume of sample, and to make the sample last for many minutes so that various experiments can be performed. Noncovalent complexes are usually analyzed by nanoESI, partly because of the small sample volumes which

Published online August 10, 2005

Address reprint requests to Dr. B. A. Thomson, MDS Sciex, Division of MDS Health Group, 71 Four Valley Drive, Concord, Ontario L4K 4V8, Canada. E-mail: bruce.thomson@sciex.com

* Also affiliated with MDS Sciex, Concord, Ontario, Canada. are typically available, but also because it is widely assumed to work better than electrospray for large complexes in aqueous solvents at low or neutral $\mathrm{pH}$, although we are not aware of any systematic experiment that has demonstrated this fact.

The defining characteristic of low flow is assumed to be responsible for the beneficial effect of high ionization and sampling efficiency, presumably because the droplets are very small and therefore can become well desolvated, given the experimental conditions employed. The overall efficiency can be determined by comparing the ion flux at the detector measured in ion counts per second at the $\mathrm{m} / \mathrm{z}$ of the sample ion with the flux of sample molecules emitted from the needle tip:

$$
\varepsilon=\frac{\text { ions } / \mathrm{sec}}{\text { samplemolecules } / \mathrm{sec}}
$$

In this equation, the denominator is calculated from the molar solution concentration and the flow rate, so that no assumption is made about whether the sample is ionized in solution. This measurement accounts for all potential losses including incomplete ionization in solution, incomplete ejection of ions from the droplets, losses in the source and through the sampling orifice, losses in the ion optics and the mass spectrometer, and losses at the detector. The use of ion counting for detection provides better accuracy and precision than the use of an electrometer, and does not require knowledge of either the ion charge or the gain of the detector. Wilm and Mann have reported efficiencies in a triplequadrupole system (equipped with a pulse-counting detector) for a peptide of 1 ion per 1300 molecules [6], and in a later report, an efficiency of 1 ion per 330 molecules [7]. In the latter study, the quadrupole resolution was reduced to improve ion transmission [7]. In both cases, the efficiency was calculated by measuring 
the flow rate and ion signal over an extended time period, and averaging both.

Geromanos et al. [8] performed a very thorough study of the characteristics of nano-electrospray using carefully controlled needle diameters, measuring sensitivity at reduced Q1 resolution as a function of flow rate and position of the needle. Reduced resolution allows the entire isotopic cluster to be transmitted, and provides higher transmission through the quadrupole and therefore higher efficiency, although at the expense of specificity. They observed both increased sensitivity and overall efficiency as the flow rate decreased to 1.6 $\mathrm{nL} / \mathrm{min}$, although below this level there was no improvement. The flow rate was varied by changing the needle diameter (i.d.) and pressure appropriate (optimum) for each flow rate, so that the results showed efficiencies when the needle diameter was optimum for each flow rate. An efficiency of $\sim 5 \%$ was observed at the lowest flow rates of 1.0 and $1.6 \mathrm{~nL} / \mathrm{min}$. The fact that the efficiency did not improve when the flow rate was reduced from 1.6 down to $1.0 \mathrm{~nL} / \mathrm{min}$ was postulated to be because all available ions were sampled at the flow of $1.6 \mathrm{~nL} / \mathrm{min}$, and further improvements may not be possible by further reducing the flow rate.

Our goal was to extend the knowledge about the absolute efficiency by conducting controlled experiments with commercially available nanospray needles to measure efficiency as a function of flow rate with a number of individual needles, varying the flow rate with fixed (although unknown) needle diameter, and to identify by means of ion current measurements where the major loss of efficiency occurs. We chose to use a quaternary ammonium salt, dodecyltrimethyl ammonium (DDTMA) bromide, as the primary model compound. Its property of high surface activity should provide high sensitivity and minimal competition from other salts in solution. Additional experiments were performed with two peptides, gramicidin $S$ and Glu- [1]-fibrinopeptide (GFB), and a protein, apo-myoglobin.

\section{Experimental}

All experiments were performed on a research test-bed identical in form to an API 3000 triple-quadrupole mass spectrometer system, using Q1 at unit mass resolution (peak width $0.7 \mathrm{Da} / \mathrm{e}$ ) for analysis, with Q3 in RF-only mode and no collision gas in Q2. Ion source parameters were adjusted for each needle as described below. The potential difference between the orifice and the skimmer, which controls the declustering, was set to $30 \mathrm{~V}$ for DDTMA and to $50 \mathrm{~V}$ for the peptides and proteins. Other ion optical parameters were not adjusted through the course of the experiments. In general, relatively high sample concentrations were employed (actual values described below), to ensure that only a single species was present, or at least predominated, in the solution so that measured ion currents could be compared with calculated fluxes of sample ions. The ion detector, located at the end of Q3, was a channel electron multiplier operated at a gain of $\sim 10^{7}$. The pulse-counting system experiences counting losses at high count rates $\left(>2 \times 10^{6} \mathrm{cps}\right)$ due to dead-time. The true ion intensities for DDTMA were determined by measuring the intensity of the third isotope at $\mathrm{m} / \mathrm{z} 230$ and then multiplying by the ratio of the intensities of the two isotopes $(228: 230=71.5: 1)$, separately determined for the experimental operating conditions at a lower count rate.

Nano-ESI needles were purchased from Proxeon Biosystems (Odense, Denmark) and from New Objective Inc (Woburn, MA). The Proxeon needles were originally closed at the tip, and were opened by gently touching the tips on the orifice plate. The New Objective needles ( $1 \mu \mathrm{m}$ tip diameter) were nominally open when received, but sometimes needed to be touched to the plate to start the flow. Both types of needles were gold-plated externally by the manufacturer to allow electrical contact to be made to the outside of the tube. In some of our earlier experiments, we used a stainlesssteel wire inserted into the tube as the electrode, but we noticed no difference between the two methods. All experimental results reported here were obtained with coated needles and electrical contact to the liquid through the metal coating. Samples were: (1) DDTMA at concentrations of $1,2,10$, and $40 \mu \mathrm{M}$ in $100 \%$ acetonitrile and in 50/50 methanol/ $0.2 \%$ formic acid; (2) apo-myoglobin at a concentration of $2.4 \mu \mathrm{M}$ in $50 / 50$ methanol $/ 0.2 \%$ formic acid; (3) gramicidin $\mathrm{S}$ at a concentration of $1 \mu \mathrm{M}$ in 50/50 methanol/ $0.2 \%$ formic acid; and (4) Glu- [1]-fibrinopeptide at concentrations of 1 and $10 \mu \mathrm{M}$ in $50 / 50$ methanol/ $0.2 \%$ formic acid.

Figure 1 shows the experimental set-up. A needle was loaded with 1-3 $\mu \mathrm{L}$ of sample, and inserted into the holder. In early experiments, the average flow rate was measured by weighing the needle before and after spraying for a set time period. However, weighing inaccuracies, evaporation losses, and the need to open the tip before spraying contributed to large experimental uncertainties. For all of the experiments reported here, a camera/monitor system with $20 \times$ magnification was used to allow the needle to be viewed against a background containing a fine wire mesh, with a measured wire spacing of $0.127 \mathrm{~mm}$. By properly positioning the camera, the liquid meniscus at the trailing edge of the liquid plug could be viewed against the mesh, and the velocity of the meniscus determined by measuring the time required to travel past a selected number of mesh cells. Multiplying by the cross-sectional area of the needle $\left(0.37 \mathrm{~mm}^{2}\right)$ allowed the volume flow to be calculated, providing near real-time measurement of the volume flow rate and correlation with the ion signal.

The flow rate was controlled by a combination of voltage and backpressure on the liquid in the needle. The pressure was controlled by using a needle valve as a bleed line, and was measured with a diaphragm manometer. Pressures of 50 to 500 torr above atmo- 


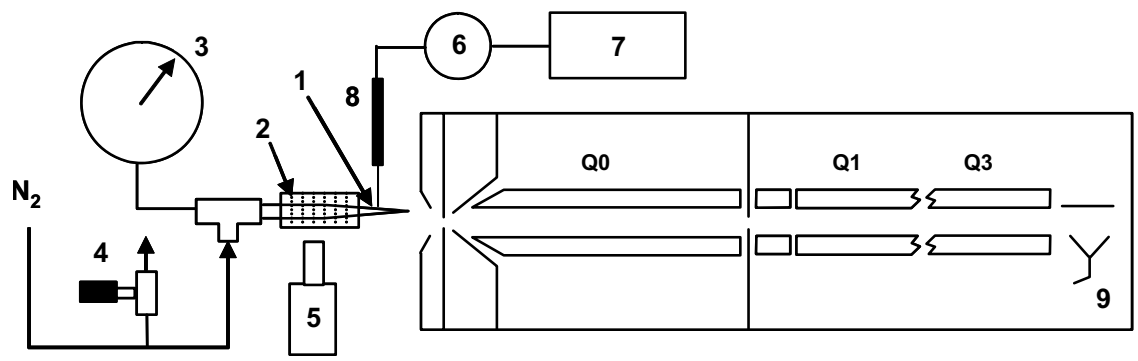

Figure 1. Nano-electrospray mass spectrometer system. 1 - Nano-electrospray needle. 2 - Grid. 3 Pressure gauge. 4 - Needle valve to control backpressure. 5 - Video camera. 6 - Picoammeter to measure needle current. 7 - High voltage power supply. 8 - $10 \mathrm{G} \Omega$ resistor. 9 - CEM connected to preamp/discriminator for pulse counting.

spheric were typically used to achieve the correct spraying conditions. After each needle was loaded and placed in the holder, the voltage and pressure were adjusted for maximum ion signal. The position of the needle was also adjustable, but it was found in general that the signal was not sensitive to minor changes in position. After establishing the spray and a stable signal (maximized for sensitivity), the averaged signal (in ion counts per second) was recorded for a period of $10 \mathrm{~s}$. The flow rate was measured by timing the movement of the meniscus over a few mesh cells (typically for 10 to $60 \mathrm{~s})$. The flow rate was then changed by increasing or decreasing the backpressure and remeasuring the flow rate and ion signal, readjusting the voltage if necessary to obtain maximum signal at each flow rate. In general, the back-pressure had a larger effect on flow rate than did the voltage.

The electric current from the needle was monitored with a custom-built picoammeter that was floated at the needle voltage. In some experiments, a $1 \times 10^{10} \Omega$ resistor was mounted between the high-voltage power supply and the needle. As Enke and Jackson [9] showed, this can prevent arcing from the needle if the voltage is increased too high, and it may also suppress corona discharge, which would contribute unwanted current to our measurements. We found no difference in the mass spectrum (above $100 \mathrm{Da} / \mathrm{e}$ ) when the resistor was used, and the spray appeared to be somewhat more stable. This is the first report of which we are aware in which a current-limiting resistor was used with a nano-ESI source.

In an effort to identify the most significant areas of ion loss, two experiments were conducted in which the current to the orifice and to lens elements in the vacuum chamber was measured with an electrometer (model 630, Keithley, Cleveland, $\mathrm{OH}$,). The current to a lens element was measured by biasing the lens with a voltage to attract all ions that reach the lens, and measuring the current under this condition. This measurement includes all of the current that would normally pass through the lens, plus the current that would normally be lost to the lens. Thus, it represents the total current that reaches the lens. As an example, the total current to the orifice was measured by reversing the field downstream of the orifice by making the skimmer potential $+100 \mathrm{~V}$ so that all ions passing through the orifice were turned back and recorded along with those lost to the orifice on the front side. This current thus represents all of the current reaching the orifice.

\section{Results and Discussion}

Many experiments were carried out over the course of several months. Impressively, there were several cases in which efficiencies of between 1 and $12 \%$ were achieved - in the best case, 1 ion detected per 8 DDTMA ions leaving the nano-ESI tip. This is more than 10 times higher than the best previously reported by Wilm and Mann [7] for a peptide (admittedly from a single experiment, in their case). Where their result was obtained with reduced mass resolution, unit mass resolution in the resolving quadrupole was maintained in our experiments. Considering the usual assumptions about losses through the orifice, the skimmer, the optics, the mass filter and the Q2/Q3 assembly, the efficiency is surprisingly high. It implies that there are some ideal conditions under which very high ionization and sampling efficiency can be achieved. To display the range of results obtained over all experiments, the results for 34 needles using DDTMA are plotted in Figure 2. This plot shows the maximum efficiency obtained for each needle, and the flow rate at which it was achieved. At these low flow rates, there is no obvious correlation between maximum efficiency and flow rate. Contained within this plot are data from four different concentrations (1, $4,10$, and $40 \mu \mathrm{M})$, from two solvent compositions (50/50 methanol/water with $0.2 \%$ formic acid, and $100 \%$ acetonitrile with no added acid or salt) and from two different needle manufacturers (Proxeon and New Objective). By separately plotting the data with common solvent compositions and common concentrations, we have found that the correlation does not improve.

However, when efficiency is plotted as a function of flow rate for one particular needle, then the trend is much clearer. It seems that each needle has its own characteristics (independent of the flow rate which can be a function of the tip diameter), and that the scatter in the data of Figure 2 is largely attributable to needle-to- 


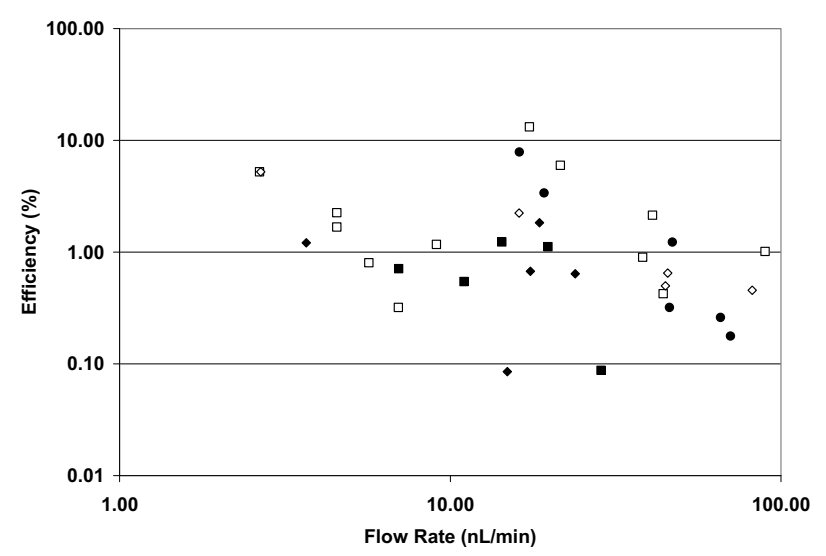

Figure 2. Maximum efficiency of 34 needles versus flow rate at which maximum efficiency was obtained. All samples were DDTMA. Each data point represents the maximum efficiency measured for one particular needle and sample, and the flow rate at which it was measured. The symbols represent the following needles and conditions: Open Square $-4 \mu \mathrm{M}$ DDTMA in MeCN. Solid Square $-10 \mu \mathrm{M}$ DDTMA in $50 / 50 \mathrm{MeOH} / 0.2 \%$ formic acid. Open Diamond - $4 \mu \mathrm{M}$ DDTMA in $50 / 50 \mathrm{MeOH} / 0.2 \%$ formic acid. Solid Diamond - $40 \mu \mathrm{M}$ DDTMA in $50 / 50 \mathrm{MeOH} / 0.2 \%$ formic acid. Solid Circle $-1 \mu \mathrm{M}$ DDTMA in MeCN.

needle variations which were beyond our experimental control. This became quite apparent during the course of the experiments, where the same sample loaded into an otherwise identical needle produced up to a factor of ten difference in ion signal, even at the same flow rate (although typical variations were less than this).

Figure 3 demonstrates this effect. Here the data from several individual needles have been gathered and plotted separately as efficiency versus flow rate. The scatter from needle-to-needle is much larger than the variability in efficiency for one individual needle. The inverse correlation is evident in every case, although the coefficients (slopes) are different. In almost every case, the best efficiency was found at the lowest flow rate.

Other samples showed similar trends, but the data are sparser. The efficiency of myoglobin $(2.4 \mu \mathrm{M}$ in $50 / 50$ methanol $/ 0.2 \%$ formic acid) was measured in two experiments. Maximum efficiencies (measured by summing all charge states, in ions per second) of $1.6 \%$ and $4.1 \%$ were measured at flow rates of 6 and 15 $\mathrm{nL} / \mathrm{min}$, respectively. The efficiencies of two peptides, gramicidin S and GFB, were also measured (summing singly and doubly charged ion signals). The maximum efficiency of gramicidin S ranged from 0.01 to $1 \%$ and that of GFB ranged from 0.1 to $0.3 \%$.

The measurements of ion current were conducted to elucidate the major areas of ion loss between the needle and the detector. It also provided an opportunity to compare the measured ion flux with the calculated molecular flux from the needle. In the absence of additional sources of ionization (electrical discharge or electrochemical) and additional salt or buffer ions, the measured ion flux, I, from the needle should be simply:

$$
I=Q n F
$$

where $Q=$ flow rate, $n=$ concentration of excess ions in the droplets, and $F=$ Faraday's constant. As an example, at a flow rate of $20 \mathrm{~nL} / \mathrm{min}\left(3.3 \times 10^{-10} \mathrm{~L} / \mathrm{s}\right)$ and 1 $\mu \mathrm{M}$ sample concentration, the emitted current should be $31 \mathrm{pA}$. This assumes the ideal case of complete ionization in solution, and that all ions in the bulk solution appear as excess charges in the droplets. In a real analytical situation, the analyte may not be fully ionized in solution, and many other ions will be present at higher concentration than the analyte. We chose a DDTMA salt so that we may be able to compare the calculated and measured ion currents, since we can reasonably expect the DDTMA to be fully ionized in solution, and that at the concentrations used, other ions are in a minority in solution. However, the electrospray process itself requires a minimum current to produce the formation and ejection of charged droplets, and additional charge carriers may be formed by electrochemical processes at the tip of the needle if the natural abundance of excess charge carriers is too low. Various efforts have been made to relate the parameters of current and flow rate. For example, Schmidt et al. found that $\mathrm{Q} \sim \mathrm{I}^{5.5}[5]$ in an investigation of flow rates up to $500 \mathrm{~nL} / \mathrm{min}$, while Fernandez de la Mora and Loscertales [10] found that $\mathrm{Q} \sim \mathrm{I}^{2}$ for electrospray, as long as the electrical conductivity of the liquid is $>10^{-5} \mathrm{~S} / \mathrm{m}$.

In our experiments, we measured charge or current balance at a flow rate of $140 \mathrm{~nL} / \mathrm{min}$ using a solution of $4 \mu \mathrm{M}$ DDTMA bromide in $100 \%$ acetonitrile. With no added acids or salts, the charge carriers should have exclusively been DDTMA cations at a concentration of 4 $\mu \mathrm{M}$. The measured ion current from the nano-ESI tip was $2.4 \mathrm{nA}$. From the equation above, the current of DDTMA ions at a flow rate of $140 \mathrm{~nL} / \mathrm{min}$ should be $0.91 \mathrm{nA}$ (assuming that the sprayed solution contained the $4 \mu \mathrm{M}$ bulk concentration of DDTMA ions), indicating that the DDTMA ions made up a maximum of $38 \%$

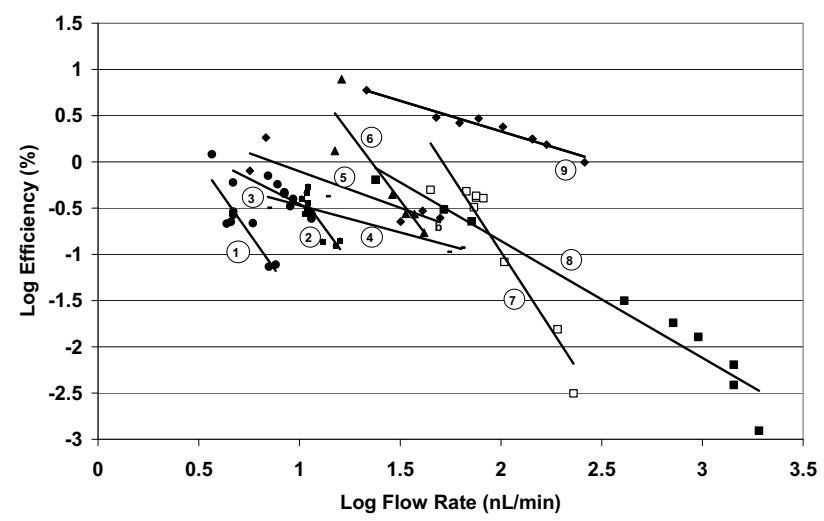

Figure 3. Efficiency versus flow rate for nine different nano-ESI needles. All samples were DDTMA in New Objective needles. 1 $40 \mu \mathrm{M}$ in MeCN. 2, $3-10 \mu \mathrm{M}$ in $50 / 50 \mathrm{MeOH} / 0.2 \%$ formic acid. $4,5,9-4 \mu \mathrm{M}$ in MeCN. $6-1 \mu \mathrm{M}$ in MeCN. $7-4 \mu \mathrm{M}$ in $50 / 50$ $\mathrm{MeOH} / 0.2 \%$ formic acid. $8-40 \mu \mathrm{M}$ in $50 / 50 \mathrm{MeOH} / 0.2 \%$ formic acid. 
Table 1. Ion currents ( $\mathrm{AA}$ ) measured from the needle to the detector

\begin{tabular}{lcc}
\hline & $\begin{array}{c}\text { Case 1 } \\
140 \mathrm{~nL} / \mathrm{min}\end{array}$ & $\begin{array}{c}\text { Case 2 } \\
20 \mathrm{~nL} / \mathrm{min}\end{array}$ \\
\hline \hline lon current from needle & 2.4 & 16 \\
DDTMA ion flux from needle* & 0.91 & 0.128 \\
lon current to orifice & 2.4 & 16.4 \\
lon current to skimmer & 1.8 & 7.5 \\
m/z 228 lon current at detector & .021 & .002 \\
$\quad$ (Q1-resolved) & 2.3 & 1.6 \\
Overall efficiency (\%) & & \\
\hline
\end{tabular}

*DDTMA ion flux is calculated from (concentration in solution) $\times$ (flowrate) assuming $100 \%$ ionization in solution. Expressed in $n A$ as calculated from eq 1.

${ }^{a}$ Case 1: $4 \mu \mathrm{M}$ DDTMA in acetonitrile

${ }^{\mathrm{b}}$ Case 2: $4 \mu \mathrm{M}$ DDTMA in 50/50 MeOH/0.2\% formic acid.

of the sprayed ion current. The additional charge carriers are likely produced from electrolysis of water, although the possibility of corona-generated ions cannot be ignored even in the presence of the $10^{10} \Omega$ resistor. Corona discharge is possible around the very small jet of liquid produced from the tip of the sprayer where the electric field strength can be high even at a voltage of only $1800 \mathrm{~V}$. For example, if the jet diameter is $1 \mu \mathrm{m}$, a voltage of $1800 \mathrm{~V}$ would produce an electric field strength exceeding $30,000 \mathrm{~V} / \mathrm{cm}$ within $30 \mu \mathrm{m}$ of the jet surface. Intermittent or pulsing gas-phase microdischarges could, therefore, contribute to the net ion current.

Table 1 shows the ion currents measured at the needle, the orifice, the skimmer, and the ion detector after mass resolution by Q1. The measured ion signal at the detector, mass-resolved in Q1 at $228 \mathrm{Da} / \mathrm{e}$, was 1.3 $\times 10^{8} \mathrm{cps}$, corresponding to a current of $0.021 \mathrm{nA}$ and an efficiency of $2.3 \%$ (0.021 nA of DDTMA, measured at the detector, compared with a calculated current of 0.91 nA of DDTMA leaving the needle, assuming complete ionization in solution). The efficiency of transmission through the orifice was $75 \%$, indicating that the ion beam (which may also have contained micro-droplets and clusters) was well directed and entrained by the gas flow into the vacuum. This efficiency is perhaps not surprising since the tip position was always optimized to produce the maximum ion current, and in general was only 2 to $3 \mathrm{~mm}$ away from the orifice. The relatively high gas flow $(\sim 0.6 \mathrm{~L} / \mathrm{min})$ through the $0.25 \mathrm{~mm}$ diameter orifice may result in the entire spray being inhaled. Such high efficiency is not expected for higher liquid flow rates ( $>1 \mu \mathrm{L} / \mathrm{min}$ ) where the spray is likely more disperse and the droplets larger in size. In another experiment, a solution $4 \mu \mathrm{M}$ DDTMA in 50/50 methanol/water containing $0.2 \%$ formic acid was sprayed, and the ion currents were measured (Case 2 in Table 1). Maximum efficiency was obtained at a flow rate of 20 $\mathrm{nL}$ per min. The flux of DDTMA ions was seven times lower than in the first case above, but the current from the needle was much higher because of the presence of formic acid in the solution-presumably, the majority of the ion current consisted of hydrated protons in the droplets. The efficiency of transmission through the orifice was $47 \%$. The overall efficiency of $1.5 \%$ for DDTMA (see Table 1) was similar to that in the first experiment, indicating that the competing presence of formic acid did not inhibit the DDTMA ion current. This is perhaps not surprising, given the high surface activity of DDTMA, which should act to significantly concentrate the ions near the droplet surface and make them very competitive for evaporation, compared with the presumably more highly hydrated protons.

The most significant losses in both cases described were, therefore, not at the atmosphere-vacuum orifice, but either in the ion source or through the ion optics and the mass filter. Although the efficiency is close to the highest previously reported for nano-ESI $(5 \%$ in reference [7], there is still a loss of $98 \%$ of the maximum available ions between the needle and the detector. The following factors could contribute to the missing ion current:

1. Ionization/desolvation efficiency of less than $100 \%$. There is no way of telling what percentage of ions in the liquid end up as free ions in the vacuum, fully desolvated. The relative absence of observable clusters in the spectrum suggests that small clusters of individual ions are not present in any observable amount, but there may still be micro-particles containing DDTMA or multimers of DDTMA [11, 12].

2. Transmission losses through the orifice, through the skimmer, Q0, interquadrupole lens, Q1 (mass resolving), Q2 and Q3 (RF-only).

3. Inefficient detection at the channel electron multiplier.

Independent experiments were conducted in which the ion current at the detector was measured with an electrometer and compared with the ion signal from the detector. These showed good correspondence suggesting that detector efficiency is at least $50 \%$, a lower limit set by the precision of our measurement. Transmission through Q1 at unit mass resolution is estimated to be at least 33\%, measured by comparing resolving to RF-only operation. Considering the measurements for the DDTMA solution in pure acetonitrile, (Case 1 in Table 1 ), the transmission efficiency through these three elements must be at least 0.75 (orifice) $\times 0.33(\mathrm{Q} 1) \times 0.5$ (detector) $=0.12$ or $12 \%$. This in fact is the same as the maximum efficiency of $12 \%$ that we measured for DDTMA. While the precision of the measurements and the estimates suggest that the equivalence is somewhat coincidental, it does seem reasonable to conclude that the variation observed in maximum efficiencies (see Figure 2), ranging from $12 \%$ down to below $0.1 \%$, is due to variations in the ion-emission and desolvation mechanism, rather than due to variations in the transmission efficiency of the remaining ion optics. The transmission efficiencies of the skimmer and the RF-only quadrupoles Q0, Q2, and Q3 are all known to be high from 
independent measurements, and total transmission loss of $50 \%$ through all four together is not an unreasonable estimate. In any event, the transmission efficiency of these elements should not vary significantly from one needle to another. Thus, the overall efficiency of the entire ion optical path, including orifice, should be indicated by the best overall efficiency measured$\sim 12 \%$. While space charge (in the form of ion-ion repulsion) could possibly affect the transmission efficiency of some elements (the skimmer, for example) from one needle to another, it seems highly unlikely that ion currents from some needles could be so large as to effectively decrease the transmitted analyte signal by up to a factor or 100. The fact that the overall efficiency for Case 2 was only marginally lower than for Case 1, even though the current from the needle was approximately six times higher, suggests that variations in ion current were not a major contributing factor to the variations in overall efficiency. While we do not claim that the transmission of the ion optics is constant for all ion sources, where the currents may be much higher, it seems likely that it was relatively constant under the experimental conditions described for our nano-electrospray experiments.

Given these results, it seems reasonable to suggest that the low efficiencies that were observed in several cases must be attributed to inefficiencies in the process of ion formation and ion sampling from the source. The most likely explanation is that the spray produced from some needles contains a large population of droplets which do not emit the ions of interest in a form that can be fully desolvated before reaching the sampling orifice This limitation on the kinetics of ion desorption could occur if the droplets evaporate too slowly (due to large initial size), or if they contain too much salt or involatile material (perhaps derived from the inner walls of the needle) to allow complete desorption of the excess analyte ions before becoming a solid residue. The initial droplet size is determined largely by the tip diameter, the voltage, the solution conductivity, and the flow rate. In many of the repeated experiments, the solutions were identical, and the only significant variation was in the individual needles. It therefore seems likely that the tip size, geometry, surface roughness, or impurities on the glass surface that become dissolved in the solution and thereby affect the conductivity or ion desorption process must be responsible for the wide variation in efficiencies.

The effect of solution conductivity, and our chosen model solutions, merit some discussion. The majority of DDTMA experiments were conducted with a low (1 to 40 $\mu \mathrm{M})$ concentration of DDTMA in acetonitrile. Fernandez de le Mora and Loscertales [10] showed that a high conductivity is required to generate small droplet sizes. It may be argued that the sample ion concentration in solution in this current study was too low to provide good spraying conditions and, in many cases, electrochemical reactions or impurities (which could vary from needle to needle) was relied upon to provide sufficient ion concentration. This could, in principle, lead to widely varying results. However, the ion current measurements shown in Table 1 indicate that in the case of DDTMA in acetonitrile, where the only ionic component in solution was DDTMA cations, the measured ion current and the calculated current (based on concentration and flow rate) were of the same order of magnitude. Clearly, no significant additional ions were created by electrochemical or other processes in this particular case. Hence, even though for many of the samples we used an unrealistically simple solution with no added salts or acid, the spray was fine and the efficiency was good. It is, therefore, unlikely that the variability we observed was due to uncontrolled (or lack of) generation of a sufficiently large number of ions from the other processes, such as corona discharge or electrochemistry. The most likely explanation seems to be that uncontrolled variations in the tip diameter, geometry, or surface roughness can affect the spray process so that the ion generation process becomes inefficient.

\section{References}

1. Iribarne, J. V.; Thomson, B. A. On the Evaporation of Small Ions from Charged Droplets. J. Chem. Phys. 1976, 64, 2287-2294.

2. Kebarle, P.; Tang, L. From Ions in Solution to Ions in the Gas Phase. Anal. Chem. 1993, 65, 972-986.

3. Juraschek, R.; Dulcks, T.; Karas, M. Nanoelectrospray-More than Just a Minimized-Flow Electrospray Ionization Source. J. Am. Soc. Mass Spectrom. 1999, 10, 300-308.

4. Karas, M.; Bahr, U.; Dulcks, T. Nano-Electrospray Ionization Mass Spectrometry: Addressing Analytical Problems Beyond Routine. Fresnius J. Anal. Chem. 2000, 366, 669-676.

5. Schmidt, A.; Karas, M.; Dulcks, T. Effect of Different Solution Flow Rates on Analyte Ion Signals in Nano-ESI MS, or: When Does ESI Turn into Nano-ESI? J. Am. Soc. Mass Spectrom. 2003, 14, 492-500.

6. Wilm, M. S.; Mann, M. Electrospray and Taylor-Cone Theory, Dole's Beam of Macromolecules at Last? Int. J. Mass Spectrom. Ion Processes 1994, 136, 167-180.

7. Wilm, M.; Mann, M. Analytical Properties of the Nanoelectrospray Ion Source. Anal. Chem. 1996, 68, 1-8.

8. Geromanos, S.; Freckleton, G.; Tempst, P. Tuning of an Electrospray Ionization Source for Maximum Peptide-Ion Transmission into a Mass Spectrometer. Anal. Chem. 2000, 72, 777-790.

9. Jackson, G. S.; Enke, C. G. Electrical Equivalence of Electrospray Ionization with Conducting and Nonconducting Needles. Anal. Chem. 1999, 71, 3777-3784.

10. Fernandez de la Mora, J.; Loscertales, I. G. The Current Emitted by Highly Conducting Taylor Cones. J. Fluid Mech. 1994, 260, 155-184.

11. Thomson, B. A. Declustering and Fragmentation of Protein Ions from an Electrospray Ion Source. J. Am. Soc. Mass Spectrom. 1997, 8, 1053-1058.

12. Ke, F.; Guevremont, R.; Siu, K. W. M. Collision-Induced Dissociation of $30 \mathrm{~m} / \mathrm{z}$ Unit-Wide Windows of ElectrosprayGenerated Ions Sampled Under Lens Conditions of Nominally Zero-Potential Gradient. J. Mass Spectrom. 1997, 32, 992-1001. 